\title{
Corrigendum: Rethinking Indian monsoon rainfall prediction in the context of recent global warming
}

Bin Wang, Baoqiang Xiang, Juan Li, Peter J. Webster, Madhavan N. Rajeevan, Jian Liu \& Kyung-Ja Ha

Nature Communications 6:7154 doi: 10.1038/ncomms8154 (2015); Published 18 May 2015; Updated 27 Jul 2015

The financial support for this article was not fully acknowledged. The Acknowledgements should have read:

\section{Acknowledgements}

We thank Professor B.N. Goswami for his constructive comments in an early version of the manuscript. This study was jointly supported by the National Natural Science Foundation of China (Grant Nos. 41420104002 and 41371209) (J. Liu and B.W.), and the Priority Academic Program Development of Jiangsu Higher Education Institutions (Grant No. 164320H116) (J. Liu), the US National Science Foundation awards \#AGS-1005599 (B.W.) and \#AGS 0965610 (P.J.W.), and the Global Research Laboratory Program from the National Research Foundation of Korea (Grant No. 2011-0021927) (K.-J.H. and B.W.). B.X. was supported by NOAA MAPP Program under Awards NA12OAR4310075. This is publication no. 9297 of the School of Ocean and Earth Science and Technology, publication no. 1108 of the International Pacific Research Center and publication no. 41 of the Earth System Modelling Center. 